\title{
Analysis of Integrate-and-Fire Neurons: Synchronization of Synaptic Input and Spike Output
}

\author{
A. N. Burkitt \\ G. M. Clark \\ Bionic Ear Institute, East Melbourne, Victoria 3002, Australia
}

A new technique for analyzing the probability distribution of output spikes for the integrate-and-fire model is presented. This technique enables us to investigate models with arbitrary synaptic response functions that incorporate both leakage across the membrane and a rise time of the postsynaptic potential. The results, which are compared with numerical simulations, are exact in the limit of a large number of small-amplitude inputs. This method is applied to the synchronization problem, in which we examine the relationship between the spread in arrival times of the inputs (the temporal jitter of the synaptic input) and the resultant spread in the times at which the output spikes are generated (output jitter). The results of previous studies, which indicated that the ratio of the output jitter to the input jitter is consistently less than one and that it decreases for increasing numbers of inputs, are confirmed for three classes of the integrate-and-fire model. In addition to the previously identified factors of axonal propagation times and synaptic jitter, we identify the variation in the spike-generating thresholds of the neurons and the variation in the number of active inputs as being important factors that determine the timing jitter in layered networks. Previously observed phase differences between optimally and suboptimally stimulated neurons may be understood in terms of the relative time taken to reach threshold.

\section{Introduction}

The integrate-and-fire model of neurons, in which the incoming postsynaptic potentials (PSPs) generate an action potential (spike) when their sum reaches a threshold, is one of the oldest (Lapicque, 1907) and most widely used models of neurons (Tuckwell, 1988a). It provides a conceptually simple description in terms of an electrical circuit in which the neural parameters (resistance and capacitance) are experimentally measurable, and it is capable of predicting interesting phenomena that can be observed in physiological experiments. A more detailed description of neurons is given in terms of nonlinear differential equations, such as the HodgkinHuxley model (Hodgkin \& Huxley, 1952), in which four nonlinear differential equations describe the membrane properties, intracellular and 
extracellular ion concentrations, input currents, and boundary-initial-value conditions. These models possess such a degree of physiological detail that in practice they are too cumbersome to address questions about the cooperative behavior of large groups of neurons. They are also deterministic and therefore do not naturally incorporate a description of stochastic (random) processes, which predominate in neural systems since the input current is rarely known with certainty. Moreover, a recent study (Kistler, Gerstner, \& van Hemmen, 1997) has shown that when a stochastic current is input to the Hodgkin-Huxley model, the spike train that is generated can be described to a very good approximation by modeling the neurons as threshold units. Networks of integrate-and-fire units thus provide models that take into account a number of the essential neurophysiological features of neurons but are still accessible with analytic techniques.

The important role that stochastic processes play in neural systems has long been recognized (see Tuckwell 1988b, 1989, for a review of stochastic processes in neuroscience). One of the earliest threshold models that incorporated stochastic inputs (Gerstein \& Mandelbrot, 1964) approximated the subthreshold potential of a spontaneously active neuron by a random walk, described by a Wiener process with drift. This model was extended (Stein, 1965) to incorporate the exponential decay of the membrane potential using stochastic differential equations. Although considerable progress has been made using these methods, there has been little progress in obtaining analytical results for more realistic models.

In this article, we present a new technique for analyzing the integrateand-fire model in the presence of stochastic synaptic input. The technique allows us to include incoming excitatory and inhibitory postsynaptic potentials (EPSPs and IPSPs, respectively) that have arbitrary time courses, so that we can incorporate such physiological features as the decay of the membrane potential and rise time of the synaptic current. A central part of the analysis is a Taylor's series expansion in the amplitude of the incoming postsynaptic potential. Only the linear and quadratic terms are retained, and consequently the technique is accurate in the limit of small-amplitude EPSPs, which necessitates a large number of inputs for the potential to reach threshold. This small-amplitude expansion enables us to calculate the probability density function of the membrane potential's reaching threshold and the probability density of output spikes, as discussed in the next section.

In the study described here, this new technique is used to examine the temporal relationship between the synaptic input and spike output of neurons for the situation where the input is synchronized within some narrow time interval, which is characterized by the standard deviation in the time of arrival (denoted as the input jitter). The situation in which the inputs are Poisson distributed can also be analyzed using similar techniques (Burkitt \& Clark, 1998a). The relationship between synaptic input and spike output is of fundamental importance to our understanding of both the coopera- 
tive processes by which neurons process information and the information contained in the neural code (Bialek, Rieke, de Ruyter van Steveninck, \& Warland, 1991; Abbott, 1994).

It is well established that the mean rate of firing of neurons plays a central role in the encoding of information in the nervous system (Adrian, 1928). However, the role played by temporal information contained in the timing of individual spikes is much less certain and has been investigated only relatively recently (Bialek \& Rieke, 1992). Part of the motivation for these studies has been the result of mathematical models of networks of spiking neurons (Abeles, 1982; Judd \& Aihara, 1993; Gerstner, 1995), which have demonstrated that the spike timing may be used in coding information (Hopfield, 1995; Gabbiani \& Koch, 1996; Maass, 1996a, 1996b). Considerable evidence indicates that the encoding of the frequency of sound in the auditory pathway uses temporal information, whereby the action potentials become locked to the phase of the incoming sound wave (see Clark, 1996, for a review of temporal coding in the auditory pathway). In the primary auditory cortex, it has been found that features of acoustical stimuli can be coded by the relative timing of action potentials of populations of neurons, even when the mean firing rate remains unchanged (deCharms \& Merzenich, 1996). Synchronization of neuronal activity on the time scale of milliseconds has been postulated to provide a mechanism by which spatially distributed cells in the visual cortex are bound together in order to represent components of a visual scene (Milner, 1974; Abeles, 1982). Recordings of the cross-correlational activity of neurons in the visual cortex have provided data that suggest that synchronization of neural activity does indeed play a functional role (for reviews, see Engel, König, Kreiter, Schillen, \& Singer, 1992; Singer, 1993).

The principal reason that synchrony of neuronal firing in groups of neurons has attracted such attention is the belief that it provides an efficient method to increase the reliability of responses: a neuron that receives many inputs simultaneously is much more likely to generate a spike than one that receives either fewer inputs or the same number of inputs distributed over a longer time interval. The importance of synchronization for neuronal information processing is on the level of groups of neurons, such as proposed by the synfire model (Abeles, 1982, 1991) in which synchronized input to a group of neurons is propagated to successive groups of neurons, called a synfire chain. Synchronization provides the possibility of establishing relationships between neuronal responses (Usher, Schuster, \& Niebur, 1993), such as grouping together (binding) neurons that respond to the same features of a stimulus (Engel et al., 1992). By establishing a synchronous firing pattern, the grouping of neurons is resistant to amplitude fluctuations, and several such assemblies of neurons can coexist. Such a mechanism would provide a neurophysiological correlate to the cognitive phenomena of scene segmentation and feature linking (Eckhorn et al., 1988; Engel, König, \& Singer, 1991). Increasing the likelihood of firing for neurons 
associated with a particular feature enables the selection of responses for further processing.

A number of theoretical studies address both the segmentation problem and the effect of synchronized inputs on the response of a single neuron (Bernander, Koch, \& Usher, 1994) or a pool of neurons (Diesmann, Gewaltig, \& Aertsen, 1996). In a recent study (Maršálek, Koch, \& Maunsell, 1997) the relationship between the spread of times of the presynaptic input and the resultant jitter of the spike output was examined using computer simulations of both the integrate-and-fire model and a detailed model of a cortical pyramidal cell. In their study, they showed that under physiological conditions, the synchronization of output spikes will be enhanced when the inputs are synchronized; that is, the output jitter will be less than the input jitter under a wide range of conditions. They also identified two sources of jitter in a cascade of such neurons as being the inhomogeneous spike propagation times between consecutive layers of neurons and jitter in the opening of the synaptic channels. In our study we show how the integrate-and-fire model may be solved analytically and give results for the perfect integrator, the Stein model, and a model that has a synaptic response function incorporating both rise time and leakage of the postsynaptic potential. The variation in threshold and the number of active inputs are both identified as also being important factors in the output jitter of a layered network. In addition, we provide an interpretation of the systematic phase difference observed between optimally and suboptimally stimulated neurons in the cat visual cortex (König, Engel, Roelfsema, Singer, 1995).

In the next section our new method for calculating the probability density of the membrane potential is presented. This technique is used to obtain the first-passage time to threshold, which is the first time that the sum of the inputs reaches threshold and therefore gives the probability density of the output spikes. The technique is then applied in section 3 to an analysis of synchronization in three integrate-and-fire neural models: (1) the perfect integrator model in which the decay of the potential across the membrane is neglected, (2) the Stein model in which each arriving EPSP gives a step increase in the potential that then decays, and (3) a model in which the incoming postsynaptic current is described by a more physiologically realistic function. In each case, the results are compared with numerical simulations. The method also enables inhibitory postsynaptic potentials to be included in a natural way, and results are given for the Stein model with inputs that are both excitatory and inhibitory. In the final section we discuss various features of the method and draw some conclusions about the results of our synchronization studies.

\section{New Method for the Analysis of Integrate-and-Fire Neurons}

Consider an integrate-and-fire neuron with a large number $N$ of incoming EPSPs, so that the resultant membrane potential at time $t$ is given by the 
sum of the inputs

$$
V(t)=v_{0}+\sum_{k}^{N} a_{k} u\left(t-t_{k}\right)
$$

where $v_{0}$ is the resting membrane potential, $N$ is the number of active inputs (i.e., number of afferent fibers that actually contribute a postsynaptic input), and $t_{k}$ is the time of arrival of the EPSP from the $k$ th fiber, which is of amplitude $a_{k}$ and has a time dependence described by the synaptic response function $u(t)$ (which has a maximum magnitude of order one). Only the case in which the amplitudes $a_{k}$ of all EPSPs have the same value $a$ is considered here. We analyze three types of integrate-and-fire models in which the neuron generates a spike when the potential reaches the threshold. We wish to calculate the relationship between the spread in the time of arrival of the inputs, characterized by the standard deviation $\sigma_{\text {in }}$ of the time of arrival of the incoming EPSPs, and the spread in the timing of the output distribution of spikes $\sigma_{\text {out }}$, also called the output jitter.

The situation we consider is where each of the incoming fibers contributes an EPSP of the same amplitude and time course and the spread in arrival times is the same for each fiber. The arrival times of the synaptic input from each fiber over many such inputs are assumed to have a gaussian distribution,

$$
p\left(t_{k}\right)=\frac{1}{\sqrt{2 \pi \sigma_{\text {in }}^{2}}} \exp \left\{-\frac{t_{k}^{2}}{2 \sigma_{\text {in }}^{2}}\right\}
$$

which has mean $t_{k}=0$ and a spread in the time of arrival of $\sigma_{\text {in }}$ (also called input jitter or width of the input distribution). We are interested in the case where the inputs produce one output spike (or equivalently, our analysis is concerned only with the first output spike generated), and refractoriness and reset effects are not included.

2.1 The Probability Distribution. In order to calculate the probability that a spike is generated, we first calculate the probability distribution of the sum $V(t)$ of the incoming EPSPs, equation 2.1. The probability that this potential $V(t)$ exceeds the value $v$ at time $t$ is evaluated by considering the proportion of cases for which this is true. This is given by integrating over the distribution of arrival times for all incoming EPSPs,

$$
\operatorname{Pr}\left\{V(t) \geq v \mid V(-\infty)=v_{0}\right\}=\left\{\prod_{k=1}^{N} \int_{-\infty}^{\infty} d t_{k} p\left(t_{k}\right)\right\} H(V(t)-v)
$$

where we assume that the membrane potential is at its resting value $v_{0}$ before the arrival of the EPSPs $\left(V(-\infty)=v_{0}\right)$. The Heaviside step function 
$H(x)$ gives a contribution of one for $V(t) \geq v$ and zero otherwise. Using an integral representation of the Heaviside step function,

$$
H\left(z-z_{0}\right)=\int_{z_{0}}^{\infty} \frac{d \lambda}{2 \pi} \int_{-\infty}^{\infty} d x \exp \{i x(\lambda-z)\}
$$

the contributions from the incoming fibers can be treated independently. Since each incoming fiber has the same distribution of arrival times of EPSPs, the above probability may be written as

$$
\operatorname{Pr}\left\{V(t) \geq v \mid V(-\infty)=v_{0}\right\}=\int_{v-v_{0}}^{\infty} \frac{d \lambda}{2 \pi} \int_{-\infty}^{\infty} d x \exp \{i x \lambda\}[F(x, t)]^{N}
$$

where the function $F(x, t)$ is given by

$$
F(x, t)=\int_{-\infty}^{\infty} d t^{\prime} p\left(t^{\prime}\right) \exp \left\{-i x a u\left(t-t^{\prime}\right)\right\}
$$

We consider the situation where the number of inputs $N$ is large and each of the inputs has an amplitude $a$ that is small (in comparison to the threshold). Expanding the exponential to second order in the amplitude $a$ of the EPSP and neglecting higher-order terms,

$$
F(x, t) \approx 1-i x a D(t)-\frac{x^{2} a^{2}}{2} E(t)
$$

where

$$
\begin{aligned}
& D(t)=\int_{-\infty}^{t} d t^{\prime} p\left(t^{\prime}\right) u\left(t-t^{\prime}\right) \\
& E(t)=\int_{-\infty}^{t} d t^{\prime} p\left(t^{\prime}\right) u^{2}\left(t-t^{\prime}\right) .
\end{aligned}
$$

The probability distribution can then be evaluated (see appendix A for details),

$$
\operatorname{Pr}\left\{V(t) \geq v \mid V(-\infty)=v_{0}\right\}=\frac{1}{2}\left[1-\operatorname{erf}\left(\frac{v-v_{0}-\Lambda(t)}{\sqrt{2 \Gamma(t)}}\right)\right],
$$

with

$$
\begin{aligned}
& \Lambda(t)=N a D(t) \\
& \Gamma(t)=N a^{2}\left(E(t)-D^{2}(t)\right) .
\end{aligned}
$$


The probability density function of $V(t)$ is given by

$$
\begin{aligned}
p\left(v, t \mid v_{0}\right) & =\frac{d}{d v} \operatorname{Pr}\left\{V(t) \leq v \mid V(-\infty)=v_{0}\right\} \\
& =\frac{1}{\sqrt{2 \pi \Gamma(t)}} \exp \left\{-\frac{\left(v-v_{0}-\Lambda(t)\right)^{2}}{2 \Gamma(t)}\right\} .
\end{aligned}
$$

In the following analysis the threshold will be expressed in terms of the threshold ratio $R$, which is ratio of the threshold $V_{\text {th }}=\theta+v_{0}$ to the maximum possible value $V_{\max }$ of $V(t)$ (the value that would be attained if all contributions arrived simultaneously), both with respect to the resting potential $v_{0}$,

$$
R=\frac{V_{\text {th }}-v_{0}}{V_{\max }-v_{0}}=\frac{\theta}{N a}
$$

where there are $N$ contributions each of amplitude $a$. We choose the units of voltage to be set by the threshold, $\theta=1$.

The expansion of equation 2.7 to second order in the amplitude $a$ of the individual EPSPs is an approximation that is good for values of $a$ that are small in comparison to the threshold. Thus, in situations where a large number of small-amplitude EPSPs are required to reach threshold, frequently the case in biological neural systems, this approximation is good. How large $N$ must be in order to provide accurate results will be examined in section 3 for a number of neural models, and the results from the analytical expressions are compared with numerical simulations. This expression for the probability density, equation 2.11, can also be obtained using the usual method of integration of the diffusion equation (Tuckwell, 1989).

2.2 Probability Density of Output Spikes. The neurons in the integrateand-fire model are endowed with a threshold condition in which a spike is generated when the summed membrane potential reaches the threshold $V_{\text {th }}$ for the first time. The probability density of the output spikes is the density of the first-passage time to threshold $f_{\theta}(t)$, that is, the probability density of the potential $V(t)$ reaching the threshold $V_{\text {th }}$ for the first time. This may be obtained from the integral equation (for $v>V_{\mathrm{th}}$ ),

$$
p\left(v, t \mid v_{0}\right)=\int_{-\infty}^{t} d t^{\prime} f_{\theta}\left(t^{\prime}\right) p\left(v, t \mid V_{\mathrm{th}}, t^{\prime}, v_{0}\right),
$$

where the function $p\left(v_{2}, t_{2} \mid v_{1}, t_{1}, v_{0}\right)$ is the conditional probability density of $V(t)$ taking the value $v_{2}$ at time $t_{2}$ given that it had taken the value $v_{1}$ at time $t_{1}$ (and also had the value $v_{0}$ at time $-\infty$ ). A similar expression has been obtained (for $v=V_{\text {th }}$ ) in a study of the response of integrateand-fire neurons to periodic input using the Ornstein-Uhlenbeck process 
(Plesser \& Tanaka, 1997). The conditional probability density may be evaluated using both the joint probability density and the probability density (see equation 2.11) via the relation

$$
p\left(v_{2}, t_{2} \mid v_{1}, t_{1}, v_{0}\right)=\frac{p\left(v_{2}, t_{2}, v_{1}, t_{1} \mid v_{0}\right)}{p\left(v_{1}, t_{1} \mid v_{0}\right)} .
$$

The joint probability density $p\left(v_{2}, t_{2}, v_{1}, t_{1} \mid v_{0}\right)$ is evaluated in a similar way to the probability density (see appendix B). In terms of the sum-over-paths formulation, the conditional probability density accounts for all paths that connect $v_{2}$ at $t_{2}$ with $v_{1}$ at $t_{1}$ (i.e., including those that are not monotonically increasing and have multiple crossings of any particular level). The resulting expression for the conditional probability density is

$$
\begin{aligned}
& p\left(v_{2}, t_{2} \mid v_{1}, t_{1}, v_{0}\right)= \\
& \frac{1}{\sqrt{2 \pi \gamma\left(t_{2}, t_{1}\right)}} \exp \left\{-\frac{\left[v_{2}-v_{0}-\Lambda\left(t_{2}\right)-\kappa\left(t_{2}, t_{1}\right)\left(v_{1}-v_{0}-\Lambda\left(t_{1}\right)\right)\right]^{2}}{2 \gamma\left(t_{2}, t_{1}\right)}\right\},
\end{aligned}
$$

where

$$
\begin{aligned}
& \gamma\left(t_{2}, t_{1}\right)=\Gamma\left(t_{2}\right)-\frac{\chi^{2}\left(t_{2}, t_{1}\right)}{\Gamma\left(t_{1}\right)} \\
& \kappa\left(t_{2}, t_{1}\right)=\frac{\chi\left(t_{2}, t_{1}\right)}{\Gamma\left(t_{1}\right)} \\
& \chi\left(t_{2}, t_{1}\right)=N a^{2}\left[G\left(t_{2}, t_{1}\right)-D\left(t_{2}\right) D\left(t_{1}\right)\right]
\end{aligned}
$$

and

$$
G\left(t_{2}, t_{1}\right)=\int_{-\infty}^{t_{1}} d t^{\prime} p\left(t^{\prime}\right) u\left(t_{2}-t^{\prime}\right) u\left(t_{1}-t^{\prime}\right)
$$

The first passage-time density may be parameterized as a gaussian distribution,

$$
f_{\theta}(t)=\frac{\rho}{\sqrt{2 \pi \sigma^{2}}} \exp \left\{-\frac{\left(t-t_{f}\right)^{2}}{2 \sigma^{2}}\right\},
$$

where $\rho$ is the probability of a spike's being produced, $t_{f}$ is the average time of the first threshold crossing (and hence time of spike production, relative to the distribution of inputs), and $\sigma$ is the jitter of the output distribution of spikes (i.e., the spread of the distribution in time), which will be labeled $\sigma_{\text {out }}$.

Equation 2.13, which defines the probability density of output spikes, is in general difficult to solve analytically. However, using the above gaussian 
parameterization (see equation 2.18) of $f_{\theta}(t)$ it is straightforward to solve for the parameters $\rho, t_{f}$, and $\sigma_{\text {out }}$ using the Newton-Raphson method for nonlinear systems of equations (see, for example, Press, Flannery, Teukolsky, \& Vetterling, 1992).

The method of solution presented here is closely related to the standard methods in which the stochastic input is modeled in terms of a random walk of the potential (Gerstein \& Mandelbrot, 1964; Tuckwell, 1988b). The case in which the inputs have a Poisson distribution has also been investigated using these methods (Burkitt \& Clark, 1998a), and the results reproduce the known expressions (Gluss, 1967). There are, however, two essential differences between equation 2.13 and the renewal equation. First, the renewal equation relates the conditional probability density to the original probability density, and this is a procedure that is valid only for a nonleaky neuron. In general there is no such relationship between the conditional probability density and the original probability density. Second, in equation 2.13 no assumptions about the stationarity of the conditional probability density $p\left(v, t \mid V_{\mathrm{th}}, t^{\prime}, v_{0}\right)$ are made; it does not have any time-translational invariance, such as occurs for the renewal equation of the nonleaky model with random inputs.

The next section presents the results for a number of neural models, which give the relationship between the spread in the time of arrival of the synaptic input $\sigma_{\text {in }}$ and the jitter of the resultant spike time $\sigma_{\text {out }}$.

\section{Synchronization in Integrate-and-Fire Neural Models}

The simplest class of models of a spiking neuron that is capable of predicting interesting experimental phenomena and in which the parameters have a physical interpretation is the integrate-and-fire model, also known as the Lapicque model (Lapicque, 1907). In these models, the arriving postsynaptic potentials simply add together until they reach threshold, at which time a spike is generated. The case in which the decay of the potential across the cell membrane is neglected is called the perfect integrator or the leakless integrate-and-fire model, which is analyzed in section 3.1. The version of the model in which the potential decays back to the resting potential is called the leaky integrator or the forgetful integrate-and-fire model. Stein (1965) was the first to analyze this model with random synaptic inputs, and the Stein model (also known as the shot-noise threshold model) is a leaky integrator model in which an incoming EPSP produces an instantaneous jump in the membrane potential, which then decays with a characteristic time constant $\tau$ (analyzed in section 3.2). We also examine (section 3.3) the leaky integrateand-fire model for the case where the synaptic response function has a physiologically realistic form that incorporates both rise time and decay.

The integrate-and-fire models are lumped (or point) models in which all the parameters of the cell are lumped together into a single representative circuit (see, for example, (Tuckwell, 1988a)). The potential difference across 
the membrane $V(t)$ is modeled by a resistor $R$ and capacitor $C$ in parallel, both of which are assumed to be constant. The input current $I(t)$ causes a depolarization of the potential $V(t)$, which, by the conservation of current, is given by

$$
C \frac{d V}{d t}+\frac{V}{R}=I(t)
$$

For subthreshold potentials, the solution of this differential equation is (Tuckwell, 1988a)

$$
V(t)=\exp \left(-\frac{t}{R C}\right) \int_{0}^{t} \frac{I\left(t^{\prime}\right)}{C} \exp \left(\frac{t^{\prime}}{R C}\right) d t^{\prime},
$$

where we assume that the cell is initially at equilibrium, $V(0)=0$. This model of the neuron is completed by imposing a threshold condition, so that when the membrane potential reaches the threshold, a spike is generated. Immediately following the spike, the membrane potential is reset to its initial value. Refractory effects may be included by allowing the threshold to become infinite immediately following the generation of a spike, corresponding to an absolute refractory period, and to have an elevated value for some limited subsequent time, corresponding to the relative refractory period.

3.1 Perfect Integrator Model. Within the family of integrate-and-fire models, the simplest case to consider is that of the perfect integrator, in which there is no decay of the potential with time. Although this is an unphysiological assumption, it may provide a reasonable approximation for situations in which the integration occurs over a time scale much shorter than the decay constant, so that the membrane potential does not decrease significantly between spikes. The model has been extensively studied because it is more amenable to analytical solution than the leaky integrateand-fire model.

In this leakless model, the individual EPSPs are each described by a simple step function:

$$
u(t)= \begin{cases}1 & \text { for } t \geq 0 \\ 0 & \text { for } t<0\end{cases}
$$

The probability density of output spikes $f_{\theta}(t)$ for this particular model may be solved exactly by considering the distribution of arrival times of the contributing EPSPs as a combinatorial problem in a manner similar to that of Maršálek et al. (1997). If the threshold is crossed with the arrival of the $M$ th input, then the resulting distribution of the output spikes is

$$
f_{M}(t)=\frac{N ! M}{M !(N-M) !} p(t) \beta^{M-1}(t)(1-\beta(t))^{N-M}
$$


where $p(t)$ is the probability distribution of incoming EPSPs (equation 2.2) and $\beta(t)$ is given by

$$
\beta(t)=\int_{-\infty}^{t} d t^{\prime} p\left(t^{\prime}\right)=\frac{1}{2}\left[1+\operatorname{erf}\left(\frac{t}{\sqrt{2} \sigma_{\text {in }}}\right)\right] .
$$

Since $p(t)$ is an even function, it follows that $f_{M}(t)=f_{N-M+1}(t)$ and hence that $\sigma_{\text {out }}(R)=\sigma_{\text {out }}(1-R)$. For large $N$, both $t_{f}$ and $\sigma_{\text {out }}$ may be evaluated by analyzing the first and second derivatives of $\ln \left(f_{M}(t)\right)$,

$$
\begin{aligned}
\frac{d}{d t} \ln \left(f_{M}(t)\right) & =\frac{t_{f}-t}{\sigma_{\text {out }}^{2}} \\
\frac{d^{2}}{d t^{2}} \ln \left(f_{M}(t)\right) & =-\frac{1}{\sigma_{\text {out }}^{2}}
\end{aligned}
$$

and thus $t_{f}$ and $\sigma_{\text {out }}$ are given by the relationships

$$
\begin{aligned}
\beta\left(t_{f}\right) & =R=\frac{M}{N} \\
\sigma_{\text {out }}^{2} & =\frac{R(1-R)}{N p^{2}\left(t_{f}\right)},
\end{aligned}
$$

where we note that the expression for $\sigma_{\text {out }}$ is again symmetric under change from $R$ to $1-R$. For small $R$, it is possible to approximate $\sigma_{\text {out }}$ as

$$
\sigma_{\text {out }}^{2}=\frac{\sigma_{\text {in }}^{2}}{-2 N R \ln R}
$$

This exact result is compared with the result obtained by the technique presented in section 2 . The functions $\Lambda(t), \Gamma(t), \gamma\left(t_{2}, t_{1}\right), \chi\left(t_{2}, t_{1}\right)$, and $\kappa\left(t_{2}, t_{1}\right)$ are given by

$$
\begin{aligned}
\Lambda(t) & =N a \beta(t) \\
\Gamma(t) & =N a^{2} \beta(t)(1-\beta(t)) \\
\gamma\left(t_{2}, t_{1}\right) & =N a^{2} \frac{1-\beta\left(t_{2}\right)}{1-\beta\left(t_{1}\right)}\left(\beta\left(t_{2}\right)-\beta\left(t_{1}\right)\right) \\
\kappa\left(t_{2}, t_{1}\right) & =\frac{1-\beta\left(t_{2}\right)}{1-\beta\left(t_{1}\right)} \\
\chi\left(t_{2}, t_{1}\right) & =N a^{2} \beta\left(t_{1}\right)\left(1-\beta\left(t_{2}\right)\right) .
\end{aligned}
$$

Since there is no inherent unit of time in this model, we choose the time scale to be set by $\sigma_{\text {in }}=1$. The results for the perfect integrator model are shown in Figure 1 for a number of inputs $N$ in the range 10 to 800 . The 
threshold ratio $R$ for a unit that sums the inputs from $N$ afferent fibers is given by equation 2.12. Both the exact solution (see equation 3.4 ) and the analytic expression (see equation 2.13) were solved for $\sigma_{\text {out }}$ at values of the threshold ratio $R=0.1,0.2, \ldots, 0.9$. The dotted lines connect the exact results of equation 3.4, and the solid lines connect the results of the numerical solution to equation 2.13. These results clearly show that the output jitter $\sigma_{\text {out }}$ decreases with increasing $N$ and that it is substantially less than the input jitter $\left(\sigma_{\text {in }}=1\right)$ over the whole range of values of $N$. The results from the analytical expression show extremely good agreement with the exact results over a wide range of thresholds for 50 inputs, and the difference diminishes for increasing $N$ such that the error is less than $1 \%$ for 100 inputs. For $N \geq 200$ the analytical results agree with the exact results over the range of threshold ratios investigated. Note that the results indicate that the minimum of $\sigma_{\text {out }}$ occurs at $R=0.5$, as expected from equation 3.7. For large numbers of inputs, the exact output spike distribution, equation 3.4, becomes gaussian, but for small $N$, there will be corrections to the gaussian parameterization, equation 2.18, which will contribute to the differences between the exact and the analytic expressions evident in Figure 1. In addition, for a fixed number of inputs, the small-amplitude approximation will be least accurate for small threshold ratios (see equation 2.12). The exact expression for $\sigma_{\text {out }}$ at small $R$, equation 3.8, is a decreasing function of $R$ because the input distribution $p(t)$ has a tail that extends to minus infinity. General considerations indicate that very low-threshold-ratio neurons tend to have high levels of spontaneous activity, whereas very high-ratio neurons tend to have very low activity and be difficult to excite. Biological neural systems would therefore be expected to function within the broad intermediate threshold region, where the technique presented here provides an accurate approximation for large numbers of input neurons.

3.2 Stein Model. Although the perfect integrator model may be adequate to explain some phenomena, it is nevertheless necessary in general to consider the effect of the leakage of the potential across the membrane. The perfect integrator model serves as a first approximation to more realistic models in which the passive membrane time constant is taken into account. Stein (1965) was the first to analyze the integrate-and-fire model with leakage of the potential in the presence of random synaptic inputs. In the Stein model the membrane potential has a discontinuous jump of amplitude $a$ on the arrival of an EPSP and then decays exponentially between inputs,

$$
u(t)= \begin{cases}e^{-t / \tau} & \text { for } t \geq 0 \\ 0 & \text { for } t<0\end{cases}
$$

where $\tau$ is the time constant of the membrane potential. The decay of the EPSP across the membrane means that the contributions from EPSPs that arrive earlier have partially decayed by the time that later EPSPs arrive. 


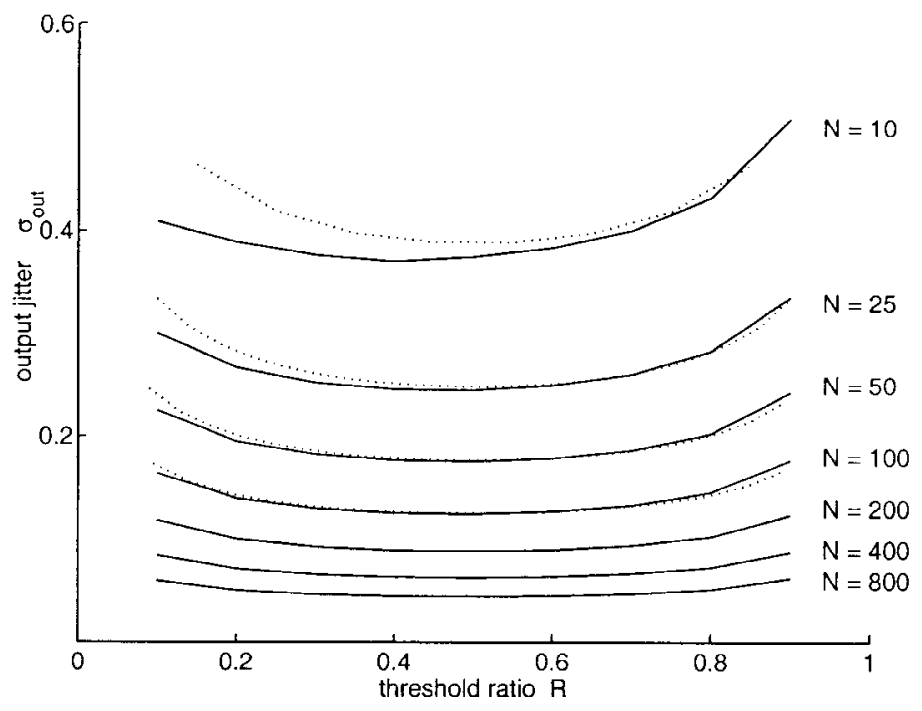

Figure 1: Results for the perfect integrator model. The dependence of the output jitter $\sigma_{\text {out }}$ on the threshold for a range of afferent fibers $N$ is shown, with $\sigma_{\text {in }}=1$. The threshold ratio $R$ is given by $\theta / \mathrm{Na}$. The solid lines connect the results of the solution to equation 2.13, and the dotted lines connect the results of the exact solution, equation 3.4. The two sets of results are indistinguishable for $N \geq 200$.

The probability density function of the potential at threshold, equation 2.11, for values of the threshold potential $v_{t h}$ that are small relative to $V_{\max }$ has a characteristic two-peak shape, as illustrated for the case of $N=50$ by the dotted and solid lines in Figure 2, which correspond to the threshold ratios $R=0.2$ and 0.4 , respectively. Time is given in units of the time constant of the membrane potential $\tau$, which typically has values of 5 to $20 \mathrm{msec}$, and the time $t=0$ corresponds to the center of the distribution of incoming PSPs, equation 2.2. Since the input jitter is typically of the order 0.5 to $3.5 \mathrm{msec}$, then $\sigma_{\text {in }}$ is small (< approximately 0.5 ). The first peak (on the left) corresponds to a net upward passage of the potential through the value $v_{t h}$ as the incoming EPSPs summate. The second peak (on the right) corresponds to the potential subsequently passing back through the same value, as there are fewer incoming EPSPs on the tail of the distribution, with the net effect being that the potential decays back to the resting value $v_{0}$. The two peaks of the probability density function merge as $R$ increases, as shown by the dashed line in Figure 2 for the case of 50 inputs with $\sigma_{\text {in }}=0.5, \tau=1.0$, and a value of $R=0.5$. At higher values of $R$, the size of the peak diminishes and eventually vanishes, which provides an effective upper limit $R_{\text {crit }}$ on 


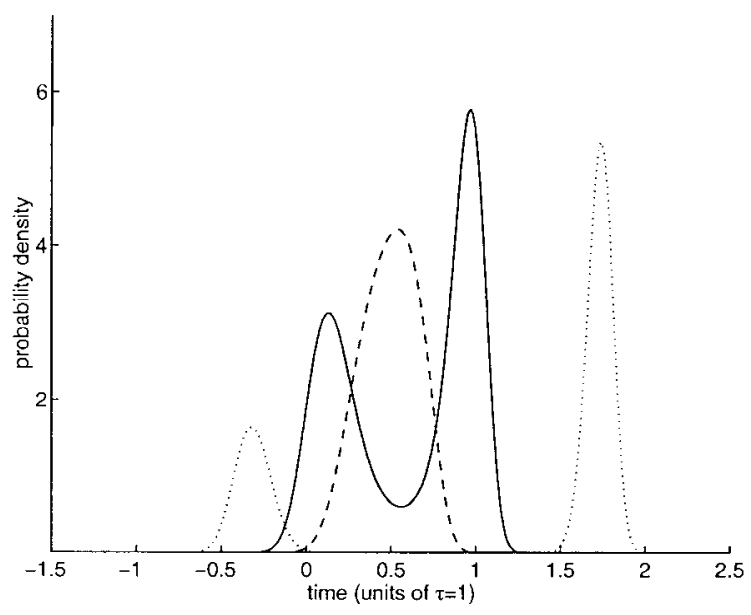

Figure 2: Representative plots of the probability density, equation 2.11, at threshold for the Stein model with 50 inputs and $\tau=1.0, \sigma_{\text {in }}=0.5$ and three values of the threshold ratio R: 0.2 (dotted line), 0.4 (solid line), and 0.5 (dashed line). Time $t=0$ corresponds to the center of the time distribution of inputs.

the threshold ratio at which a spike can be generated. For large numbers of inputs $N$, the width of both peaks decreases.

For a unit that sums the inputs from $N$ afferent fibers, the maximum possible value of the potential $V(t)$ if all inputs were to arrive simultaneously would be $V_{\max }=v_{0}+\mathrm{Na}$, and the threshold ratio $R$ is given by equation 2.12, as for the perfect integrator model. As the threshold ratio increases, the probability of an output spike's being generated falls towards zero. This dependence of the spiking probability, $\rho$, on the threshold ratio, $R$, is illustrated in Figure 3, which shows this relationship for a value of the input jitter $\sigma_{\text {in }}=0.2$ and for various numbers of inputs $N=25,100,800$. The plot shows that for low-threshold ratios, $R$, an output spike is generated with probability 1 , and that for large values of $R$, the spiking probability decreases rapidly toward zero. The rate of decrease of the firing probability depends on the number of inputs, with a more rapid falloff observed for larger numbers of inputs. Note that the threshold ratio, $R$, is defined in a way (see equation 2.12) that relates the number and magnitude of the incoming PSPs. The value of the threshold ratio $R_{\text {crit }}$ at which the spiking probability falls to zero also depends on both the number of inputs $N$ and the input jitter $\sigma_{\text {in, }}$ as plotted in Figure 4 . The results show that the effective maximum that the potential achieves depends on the input jitter $\sigma_{\text {in, }}$ with more input jitter lowering the maximum value attained by the potential. The criteria used 


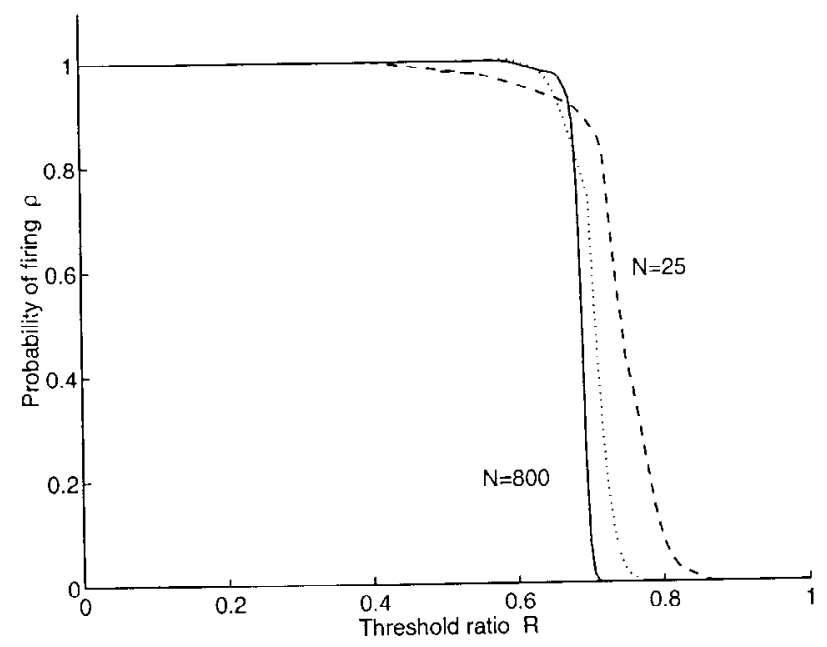

Figure 3: Probability of an output spike being generated, $\rho$, as a function of the threshold ratio, $R$, for the Stein model with input jitter $\sigma_{\text {in }}=0.2$ (in units of the membrane time constant, $\tau=1$ ). Results for three different values of $N$ are plotted: $N=800$ (solid line), 100 (dotted line), and 25 (dashed line).

for determining $R_{\text {crit }}$ in Figure 4 were

$$
\max _{t} \int_{V_{\mathrm{th}}}^{\infty} d v p\left(v, t \mid v_{0}\right) \leq 0.01
$$

A dependence of $R_{\text {crit }}$ on the number of inputs $N$ can be seen in Figure 4, which is a consequence of the probability distribution $p\left(v, t \mid v_{0}\right)$ being less sharply peaked for smaller values of $N$. It is straightforward to calculate $R_{\text {crit }}$ in the large $N$ limit, since the system is essentially deterministic in this limit; the resulting values are plotted in Figure 4 as triangles on the vertical axis. Also shown in Figure 4 for comparison are the results of numerical simulations at the value $\sigma_{\text {in }}=0.5$ for the probability of spike generation falling below 0.01 (note that the probability distribution of the potential at threshold and the probability distribution of spike generation are not identical, although they are related for large $N$, as discussed in Section 4).

The relative output jitter (i.e., the ratio of the output jitter $\sigma_{\text {out }}$ to the input jitter $\sigma_{\text {in }}$ ) is plotted in Figure 5 for $\sigma_{\text {in }}=0.2$ and a range of values of threshold ratios $R$ and inputs $N$. The integral equation 2.13 for the output spike density was solved numerically, using the Newton-Raphson method as before, for a range of threshold ratios below $R_{\max }, R=0.10,0.15, \ldots, 0.55$, and the 


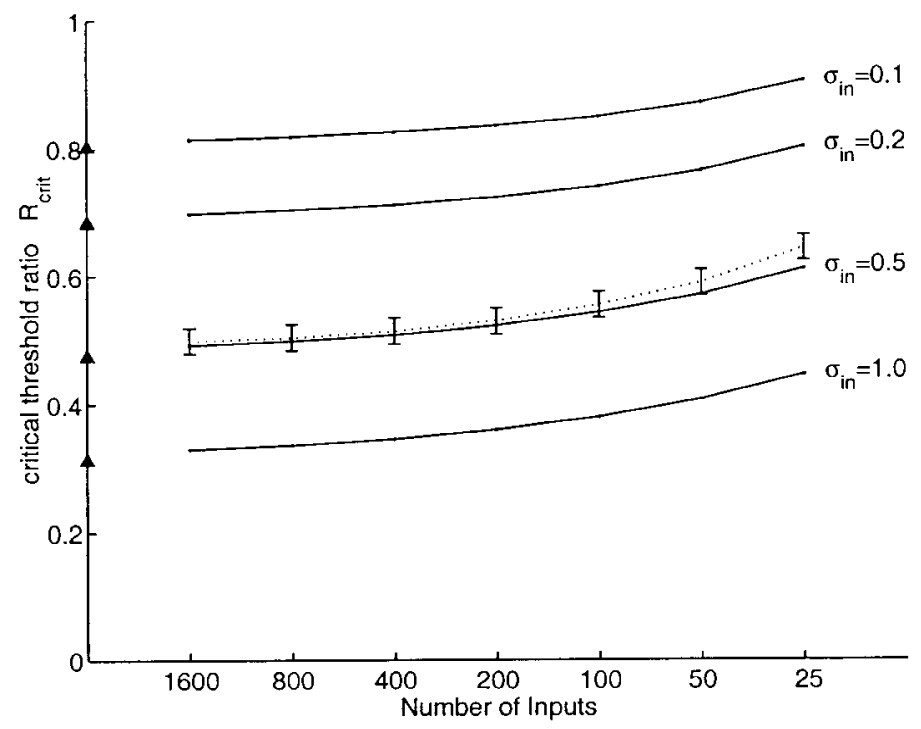

Figure 4: Dependence of $R_{\text {crit }}$ on the number of inputs $N$ and the jitter of the input $\sigma_{\text {in }}$ for the Stein model (units of $\tau=1$ ). The lines connect points at which the probability density, equation 2.11, obeys the criteria equation, 3.11. Also shown for comparison are the results with $\sigma_{\text {in }}=0.5$ of the numerical simulations for the probability of spike generation falling below 0.01 . The exact results for the large $N$ limit are indicated by triangles on the vertical axis.

results are connected by the solid lines. Also plotted are the results of a number of numerical simulations, each point representing the average over 10,000 trials. In these simulations, a gaussian distribution of arrival times for the $N$ inputs was generated using a pseudorandom number generator, and the potential was summed explicitly, taking into account the decay constant $\tau$. The error bars give the standard deviation over the trials, and the results for each value of $N$ are connected by a dashed line (for the larger values of $N$, the error bars are roughly the width of the lines and therefore are barely discernible). The relative output jitter is clearly substantially less than the input jitter over the whole range of inputs and threshold ratios investigated. The results from the analytical expression derived here are very accurate for large numbers of inputs $N$, as shown by their closeness with the results of the numerical simulations. As before, the expected error of the method presented here decreases as the number of inputs $N$ increases and the amplitude $a$ of each individual contribution decreases.

As the input jitter $\sigma_{\text {in }}$ becomes smaller relative to the membrane time constant $\tau$, the importance of the decay of the potential across the membrane diminishes and the results are increasingly well approximated by the perfect 


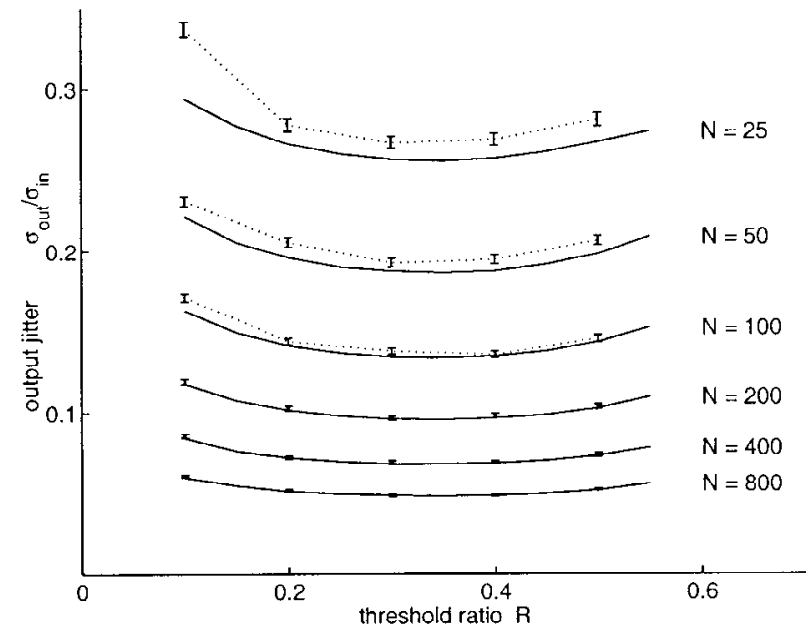

Figure 5: Relative output jitter $\sigma_{\text {out }} / \sigma_{\text {in }}$ for the Stein model with various numbers of input EPSPs and threshold ratios $R$. The jitter of the input $\sigma_{\text {in }}$ is 0.2 in units of the membrane time constant $(\tau=1)$. The solid line shows the value obtained from the solution of equation 2.13, and the data points connected by the dotted lines are each the result of 10,000 numerical simulations.

integrator model (see section 3.1). This is illustrated in Figure 6, in which the ratio of the output jitter to the input jitter is plotted for a threshold ratio of $R=0.25$ and various numbers of inputs. Plotted on the vertical axis as triangles are the results of the perfect integrator model, in which there is no decay of the potential across the membrane (equivalent to the limit of large $\tau$ ). The plots for a given number of inputs $N$ show only a very slight dependence on the ratio of the input jitter to the membrane time constant over the range of values investigated, and the results in the limit $\sigma_{\text {in }} / \tau \rightarrow 0$ extrapolate smoothly to the results of the perfect integrator model.

The effect of inhibitory postsynaptic potentials (IPSPs) may be included in a straightforward way. The functions $\Lambda$ and $\Gamma$ of equation 2.10 become

$$
\begin{aligned}
& \Lambda(t)=N_{E} a_{E} D_{E}(t)-N_{I} a_{I} D_{I}(t) \\
& \Gamma(t)=N_{E} a_{E}^{2}\left(E_{E}(t)-D_{E}^{2}(t)\right)+N_{I} a_{I}^{2}\left(E_{I}(t)-D_{I}^{2}(t)\right),
\end{aligned}
$$

where $D_{E, I}$ and $E_{E, I}$ are given by equation 2.8 for the excitatory and inhibitory neurons, respectively. The amplitudes of the excitatory and inhibitory inputs are denoted by $a_{E}$ and $a_{I}$, respectively (for simplicity in the analysis below, we choose them to be equal, $a_{E}=a_{I}=a$ ). The effects 


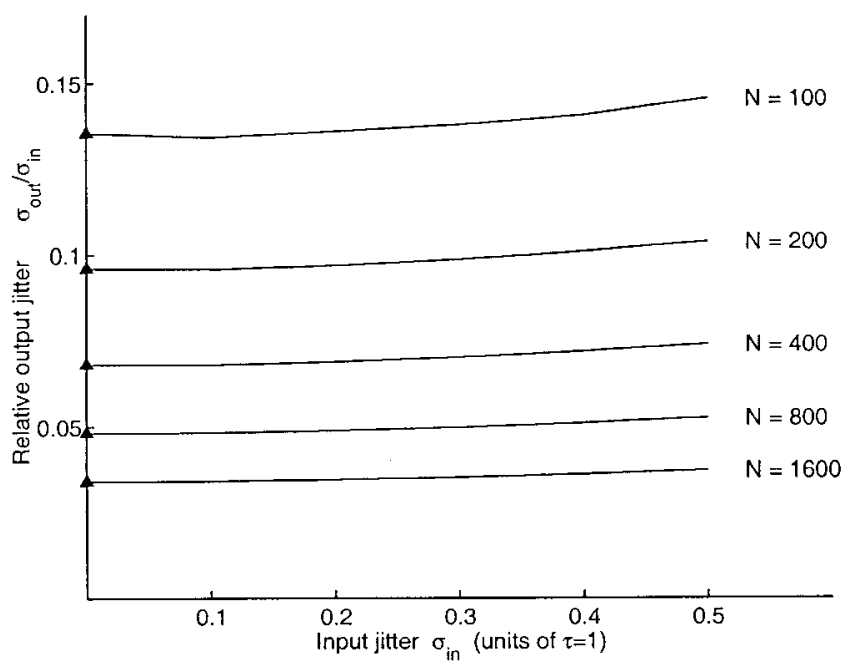

Figure 6: Relationship between the relative output jitter $\sigma_{\text {out }} / \sigma_{\text {in }}$ and the input jitter $\sigma_{\text {in }}$ for the Stein model with threshold ratio $R=0.25$ and varying numbers of inputs. The jitter of the input $\sigma_{\text {in }}$ is given in units of the membrane time constant $(\tau=1)$. The results for the perfect integrator model are indicated by triangles on the vertical axis.

of including IPSPs in the Stein model are shown in Figure 7, in which the relative output jitter is plotted as a function of the proportion of IPSPs to EPSPs, for the case $\sigma_{\text {in }}=0.2$ (in units of $\tau=1$ ). The threshold ratio in Figure 7 is fixed at the value $R=0.25$, where $R$ is defined in the analogous way to equation 2.12,

$$
R=\frac{\theta}{\left(N_{E}-N_{I}\right) a} .
$$

Note that for fixed $R$, the amplitude of the individual postsynaptic potentials increases as $N_{I}$ increases, which ensures that the increase in relative output jitter with increasing $N_{I}$ observed in Figure 7 is not an artifact of changing the range of the potential relative to a fixed threshold. Also shown in the figure are the results of numerical simulations for the cases $N=50,100,200$, each point representing the average over 10,000 trials, with the accuracy indicated by error bars. As before, the simulation results agree well with the analytical results for large $N$. We also examined the case where the amplitude $a$ is fixed and the threshold ratio increases as $N_{I}$ increases. The results in this case showed the same pattern as in Figure 7, with an increase in the relative output jitter of the same magnitude for each value of $N_{I}$, 


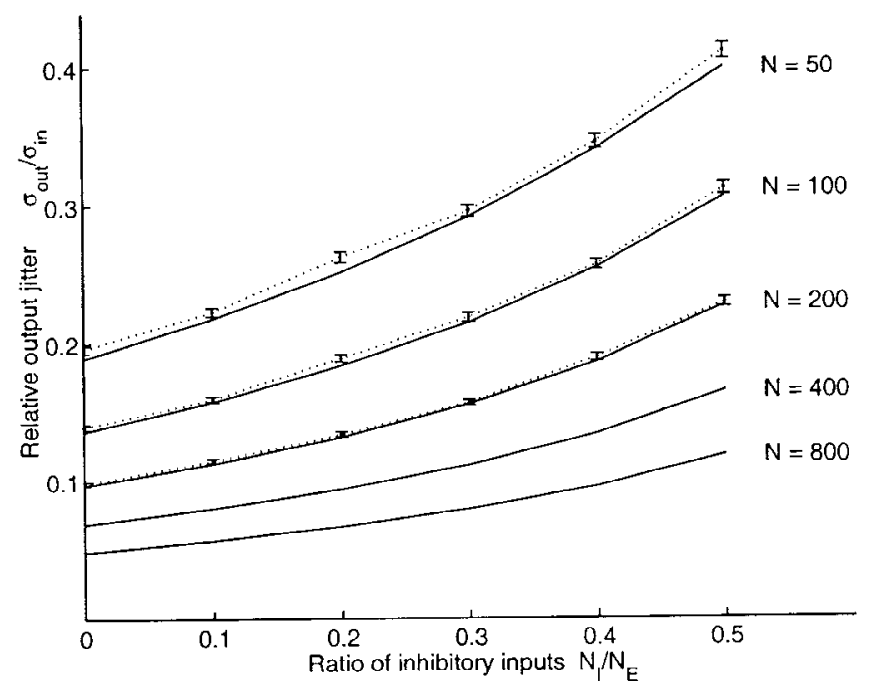

Figure 7: Relationship between the relative output jitter $\sigma_{\text {out }} / \sigma_{\text {in }}$ and the proportion of inhibitory inputs for the Stein model with input jitter $\sigma_{\text {in }}=0.2$ (in units of $\tau$ ) and fixed threshold ratio $R=0.25$. The magnitudes of the amplitudes of the excitatory and inhibitory inputs are taken to be the same, and the threshold ratio is given by equation 2.12, as described in the text. Also plotted are the results of numerical simulations over 10,000 trials for $N_{E}=50,100,200$.

indicating that the increase is indeed due to the inhibition rather than the parameterization. The results in Figure 7 are for the situation where both the amplitudes and the postsynaptic functions of the EPSPs and IPSPs are the same. However, the technique can be used equally well in the situation where the EPSPs and IPSPs have different time courses and amplitudes. It is also possible to extend the analysis to include reversal potentials (Burkitt \& Clark, 1998b).

3.3 General Leaky Integrate-and-Fire Model. Both the perfect integrator model and the Stein model have discontinuous voltage trajectories; there is an instantaneous jump of amplitude $a$ in the voltage when the EPSP arrives. A smoothly varying voltage similar to that observed in intracellular recordings is provided by a synaptic input current whose time course is given by the alpha function (Jack, Noble, \& Tsien, 1985),

$$
I(t)=k t e^{-\alpha t}, \quad \alpha>0,
$$




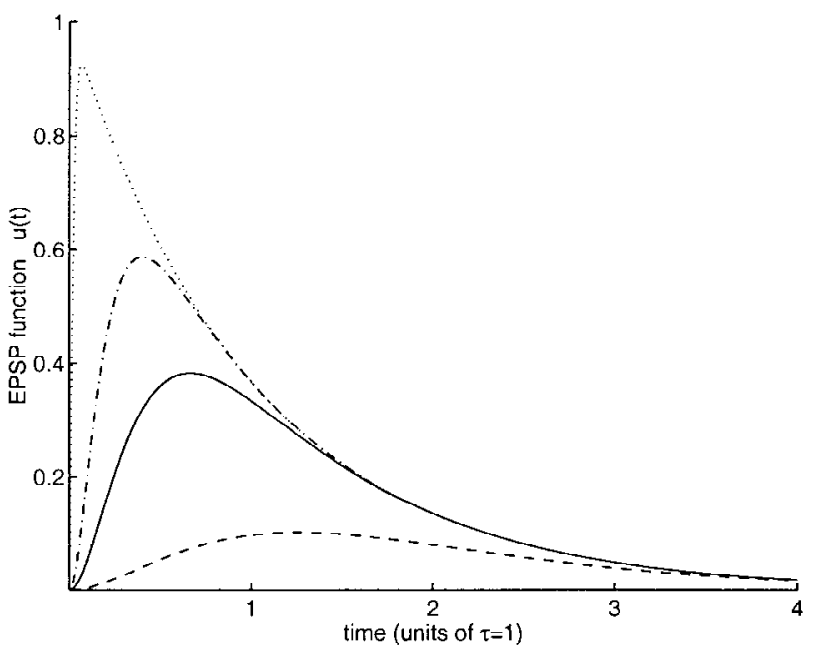

Figure 8: Synaptic response function $u(t)$ (see equation 3.15) for the general leaky integrate-and-fire model for input currents of the form of an alpha function (see equation 3.14) with $k=B^{2} C, \tau=1$. Plots are shown for values of $\alpha$ of 2 (dashed line), 5 (solid line), 10 (dash-dot line), and 100 (dotted line).

which corresponds to delivering a total charge of $k / \alpha^{2}$ to the cell. The synaptic response function $u(t)$ is, from equation 3.2 and assuming $u(0)=0$,

$$
\begin{array}{ll}
u(t)=\frac{k e^{-t / \tau}}{B C}\left[t e^{B t}-\frac{\left(e^{B t}-1\right)}{B}\right], & B \neq 0 \\
u(t)=\frac{k t^{2}}{2 C} e^{-t / \tau}, & B=0,
\end{array}
$$

where $\tau=R C$ and $B=1 / \tau-\alpha$. A plot of $u(t)$ is given in Figure 8 for $k=B^{2} C$ and four values of $\alpha$, which shows the nonzero rise time and exponential decay of a EPSP that is evident in intracellular potential recordings (Rhode \& Smith, 1986; Paolini, Clark, \& Burkitt, 1997). The Stein model is recovered in the limit $\alpha \rightarrow \infty$. Models such as this that have a finite rise time provide an approximation of the postsynaptic potential at the soma (or specifically at the site at which the action potential is generated) that incorporates the time course of the diffusion of the current along the dendritic tree (Tuckwell, 1988a).

Figure 9 shows the relative output jitter for the general leaky integrateand-fire model with $\alpha=5$ and $\sigma_{\text {in }}=0.2$, where time is measured in units of the membrane time constant $\tau$. The value of $\alpha$ determines the rise time 
of the synaptic input current, which achieves its maximum at time $t=1 / \alpha$. The value $\alpha=5$ is therefore somewhat on the low side of physiologically realistic values, but was chosen in order to contrast the results with those of the Stein model (which has zero rise time, $\alpha=\infty$ ). In order to provide a direct comparison with the results for the Stein model, the threshold ratio is defined to be the same: $R=\theta / N a$. (Note, however, that the maximum possible value of $V(t)$ that could be attained if all contributions arrived simultaneously is $V_{\max }=v_{0}+N a u_{\max }$ and that $u_{\max }=0.3826$ for $\alpha=5$.) The analytical expression (see equation 2.13) was numerically solved for $R=0.05,0.1,0.15,0.2,0.25,0.3$, and the results for each value of $N$ are connected by the solid lines in Figure 9. The error bars give the results obtained from 10,000 numerical simulations, as described in the previous section (again the error bars are barely discernible for the larger values of $N$ since they are of the same magnitude as the width of the lines). The critical value of the threshold ratio $R$, above which no output spikes are generated, range from 0.392 (for $N=25$ ) to 0.363 (for $N=800$ ) for the particular model parameters in Figure 9. The results, which are slightly lower than for the Stein model (see Figure 5), again indicate that the relative output jitter is substantially less than one over the whole range of thresholds and inputs studied and that it decreases with an increasing number of inputs. The results also show excellent agreement with the numerical simulations for large numbers of inputs, again indicating that the small-amplitude approximation required for the analytic expression is extremely accurate even for quite modest values of $N$.

\section{Discussion and Conclusions}

In this study we have presented a new method for analyzing integrate-andfire neurons with a large number of small-amplitude inputs. This technique allows the analysis of models with arbitrary synaptic response functions, in particular models that incorporate both leakage and a finite rise time of the postsynaptic potential, which has previously been possible in only very restricted cases. The method has been used to examine the question of the relationship between the temporal dispersion of synchronized inputs and the resulting jitter of the spikes that are generated. The analytic method presented here gives the output spike distribution in terms of an integral equation. The first three moments of this distribution, which give, respectively, the probability of an output spike, the average time of spike generation, and the output jitter, are solved using standard numerical techniques. The results are compared with the exact solution for the perfect integrator model and with numerical simulations for the Stein model and a model that includes both the membrane time constant and the current rise time. The computational resources required for the numerical simulations increase with the number of inputs and the required numerical accuracy, 


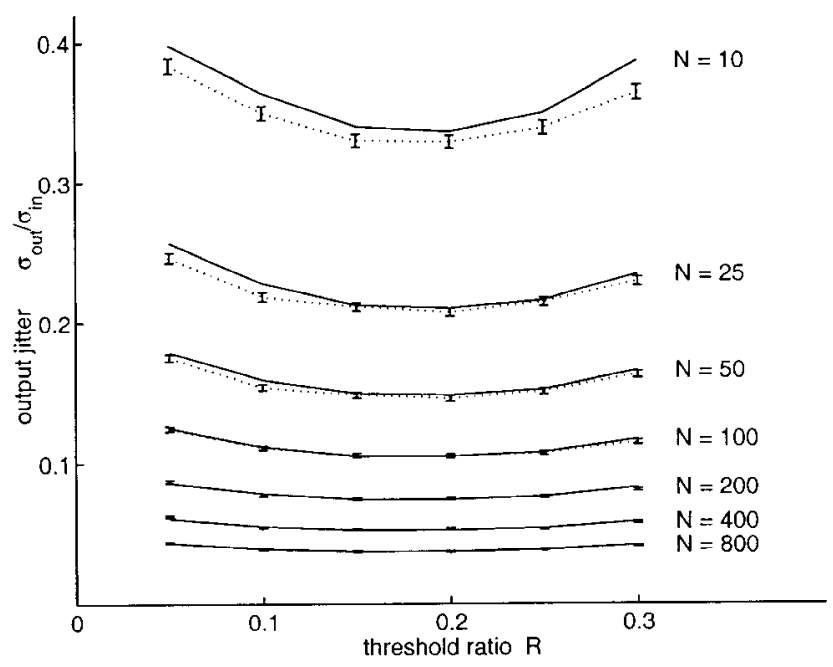

Figure 9: Relative output jitter $\sigma_{\text {out }} / \sigma_{\text {in }}$ for the general leaky integrate-and-fire model with $\alpha=5$, various numbers of inputs, and a range of threshold ratios $R=\theta / N a$. The jitter of the input $\sigma_{\text {in }}$ is 0.2 in units of the membrane time constant $(\tau=1)$. The solid line connects values obtained from the solution of equation 2.13, and the data points with error bars are each the result of 10,000 numerical simulations.

and are typically many orders of magnitude larger than those required for the numerical solution of the analytical equation.

The analytical method, which is exact in the limit of a large number of small-amplitude inputs, is shown to provide an accurate solution when the number of inputs exceeds the order of 100 . This technique allows the analysis of the whole class of integrate-and-fire neural models, from the simple perfect integrator to models that incorporate important physiological features, and therefore it can be used to test and analyze a wide variety of neural phenomena. Integrate-and-fire models form an important bridge between simpler neural models, which may have unrealistic approximations, and full-scale computational simulations of particular cells, which frequently require massive computational resources and in which the results may be specific to the cells studied. Thus, the models presented here represent a compromise between the opposing goals of neurophysiological detail and analytical transparency.

Since the individual inputs add linearly, it is possible to include a variety of synaptic response functions in equation 2.1 in order to model the effect of synapses at different parts of the synaptic tree (i.e., synapses nearer 
the soma having a synaptic response function with larger amplitude and shorter rise time than synapses farther away). The technique also enables a distribution of PSP amplitudes to be analyzed, including amplitudes that fluctuate randomly about a mean value, which could be used to model the effect of quantal fluctuations. Furthermore, it would be possible to examine the situation in which the excitatory and inhibitory inputs have different distributions, such as when inhibitory inputs arrive later than excitatory inputs. The technique has been used to examine integrate-and-fire neurons with Poisson-distributed inputs, as well as examine inputs in which the amplitudes $a_{k}$ (see equation 2.1) of the synaptic response functions have a distribution of values (Burkitt \& Clark, 1998a).

The results of the analysis of the relationship between the input jitter and the output jitter provide clear support for earlier studies (Bernander et al., 1994; Diesmann et al., 1996; Maršálek et al., 1997) showing that the jitter of the spike output is much less than the jitter on the incoming PSPs; the temporal dispersion of the output spikes is less than the temporal dispersion of the inputs, $\sigma_{\text {out }}<\sigma_{\text {in }}$, over a wide range of physiologically realistic conditions. Such a reduction in the temporal jitter has indeed been observed experimentally in the anteroventral cochlear nucleus (Joris, Carney, Smith, \& Yin, 1994). In this study the synchronization (or phase locking) to lowfrequency acoustic tones was measured for both auditory nerve fibers and cells in the anteroventral cochlear nucleus, the first stage of processing in the auditory pathway. The synchronization coefficient (Johnson, 1980) of cells in the output tract of the anteroventral cochlear nucleus was found to be enhanced relative to the incoming auditory nerve fibers. This provides evidence that a reduction in temporal jitter is possible in the nervous system.

The relationship of the relative output jitter $\sigma_{\text {out }} / \sigma_{\text {in }}$ to the number of inputs $N$ is of particular interest, especially in the large $N$ limit, and this is illustrated in the log-log plot of Figure 10. This figure shows plots for the exact solution of the perfect integrator model (shown by the solid line with triangles for $R=0.5$ and $\sigma_{\text {in }}=0.2$ ) and the analytical solution of the Stein model (the dotted line with squares shows the results for $R=0.3$ and $\sigma_{\text {in }}=0.2$ ), the Stein model including inhibition (the dash-dot line with diamonds is the case $N_{I} / N_{E}=0.5, R=0.25$, and $\left.\sigma_{\text {in }}=0.2\right)$, and a general leaky integrate-and-fire model (the dashed line with circles are the results for $\alpha=5, R=0.15$ and $\sigma_{\text {in }}=0.2$ ). All plots have a slope of $-1 / 2$ for large $N$, indicating that the width of the output spike distribution decreases with $1 / \sqrt{N}$ for large $N$ for all three models (for fixed threshold ratio $R$ ). The effect of including both the time constant of the membrane and a rise time for the synaptic response function is, for physiologically realistic values, found to be relatively small. Including inhibitory postsynaptic potentials, however, is found to cause a larger increase in the jitter of the output spikes, in agreement with earlier studies (Maršálek et al., 1997). It is also interesting to note that the three plots in Figure 10 in which only excitatory inputs 


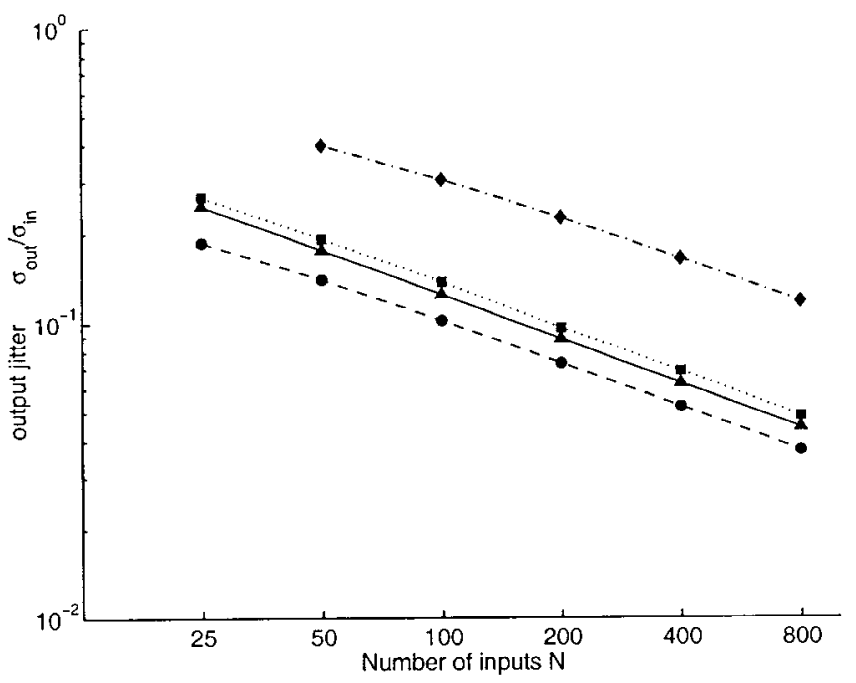

Figure 10: Log-log plot of the dependence of the relative output jitter $\sigma_{\text {out }} / \sigma_{\text {in }}$ on the number of inputs $N$. The solid line with triangles is the perfect integrator model with $R=0.5$ and $\sigma_{\text {in }}=0.2$; the dotted line with squares is the Stein model with $R=0.3$ and $\sigma_{\text {in }}=0.2$; the dashed line with circles is the general leaky integrate-and-fire model with $\alpha=5, R=0.15$, and $\sigma_{\text {in }}=0.2$; the dash-dot line with diamonds is the Stein model with inhibition $N_{I} / N_{E}=0.5, R=0.25$, and $\sigma_{\text {in }}=0.2$.

are included have numerical values that are very close, indicating that the values of $\sqrt{N} \sigma_{\text {out }} / \sigma_{\text {in }}$ are almost identical (the values are also found to have only a small dependence on the value of the threshold ratio $R$ ).

As the number of inputs increases, it is also of interest to compare the probability density function of the membrane voltage with the probability density of output spikes. For models that include a membrane decay term, the probability density function $p\left(v, t \mid v_{0}\right)$ has a characteristic two-peak structure, as discussed in section 3.2 in relation to the Stein model. The first peak of the probability density function at the threshold $p\left(V_{\mathrm{th}}, t \mid v_{0}\right)$ is expected to have an average and width of distribution that closely approximates that of the probability density of output spikes $f_{\theta}(t)$, since it corresponds to the upward passage of the potential through the threshold. As the number of inputs $N$ increases, the peaks of the probability density function at the threshold $p\left(V_{\mathrm{th}}, t \mid v_{0}\right)$ become increasingly sharp; for threshold ratios $R$ below the critical threshold ratio $R_{\text {crit, }}$, this approximation to $f_{\theta}(t)$ by the first peak improves. This is shown in Figure 11, where the ratio of the width of the probability density distribution $\sigma_{\mathrm{pd}}$ at threshold and the width 


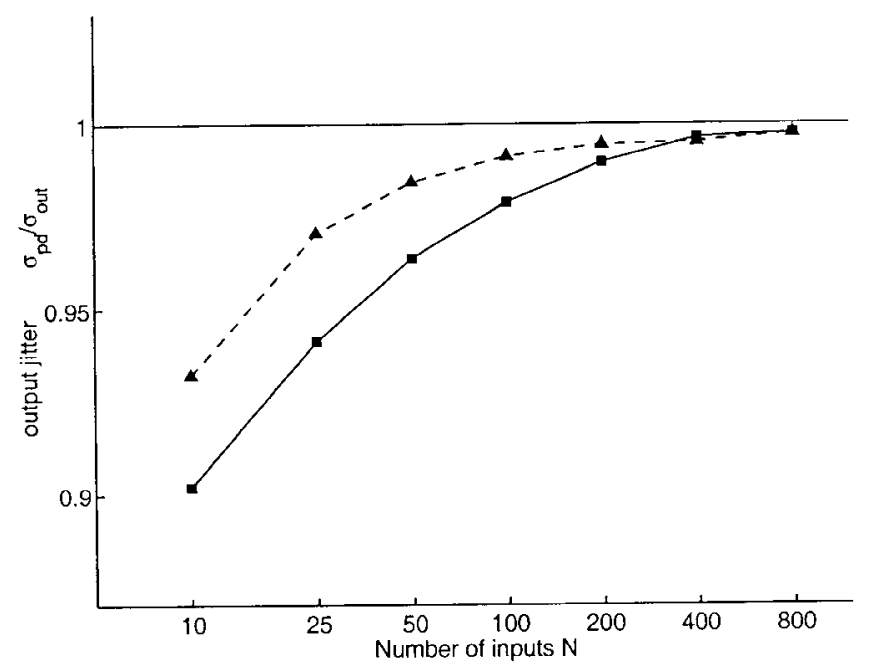

Figure 11: Ratio of the width of the probability density to the output jitter $\sigma_{\text {pd }} / \sigma_{\text {out }}$ as a function of the number of inputs $N$ for the leaky integrate-andfire model with $\alpha=5$ and $\sigma_{\text {in }}=0.2$. The solid line with squares shows the results for $R=0.1$, and the dashed line with triangles shows the results for $R=0.2$.

of the spike output distribution $\sigma_{\text {out }}$ is plotted as a function of the number of inputs $N$ for the general leaky integrate-and-fire model of section 3.3 with $\alpha=5, \sigma_{\text {in }}=0.2$ and $R=0.1$ (solid line with squares), and $R=0.2$ (dashed line with triangles). The results show that $\sigma_{\mathrm{pd}}$ converges toward $\sigma_{\text {out }}$ and that $\sigma_{\text {pd }}$ therefore provides an approximation to $\sigma_{\text {out }}$, which becomes increasingly accurate for large values of $N$. However, the equivalence is not exact (the ratio is not exactly 1) since the probability density of the potential contains information about multiple threshold crossings. A plot of the conditional probability density $p\left(V_{\mathrm{th}}, t \mid V_{\mathrm{th}}, t^{\prime}, v_{0}\right)$ shows a large peak at $t=t^{\prime}$, corresponding to the first threshold crossing (this would actually be a delta function if $p\left(V_{\mathrm{th}}, t \mid v_{0}\right)$ was equal to $f_{\theta}(t)$, as is evident from equation 2.13) followed by a tail, corresponding to the multiple crossings, and a second more rounded peak, corresponding to the second peak in $p\left(V_{\mathrm{th}}, t \mid v_{0}\right)$ from the passage of the voltage back to the resting value after the burst of inputs.

In a cascade of neurons, there are a number of sources of variability, in addition to the jitter of the inputs, that determine the stability of the neuronal firing pattern (Gerstner \& van Hemmen, 1996) and prevent the output jitter from converging to zero. Maršálek et al. (1997) identified two important 
factors that introduce timing variability to the arriving PSPs: the delay due to different spike propagation times and the jitter associated with the synapses. Another important factor is the variation in the spiking thresholds of the neurons, which will cause different neurons to spike at different relative times. This variability is illustrated in Figure 12, which shows the average times of spiking (measured relative to the center of the incoming distribution of arrival times, taken to be $t=0$ ) for different threshold ratios and numbers of inputs. There is only a small variation in the average time of the output spikes for neurons with the same threshold ratio $R$ (values given at the top of Figure 12) and different numbers of inputs $N$. There are, however, substantial differences in the average times at which spikes are generated by neurons with different threshold ratios. Consequently, variations of the spiking threshold over a layer of neurons will cause variations in the relative timing of the output spikes produced by the population of neurons. In such a layered network, this variation in the timing of the spikes from the previous layer will represent jitter on the inputs to the subsequent layer, which is additional to the inherent jitter associated with the production of the spikes.

Equivalently, the results presented in Figure 12 indicate that if the neurons in a particular layer have varying numbers of active inputs, then their relative times of firing will depend crucially on their number of inputs. Neurons with the same absolute threshold $V_{\text {th }}$ but fewer active inputs $N$ have a larger threshold ratio, as defined by equation 2.12. The results in Figure 12 therefore predict that units with the same absolute threshold but fewer active inputs will have a relative lag in their response. Such a phase lag has been reported in a study of the temporal relationship between responses of optimally and suboptimally stimulated neurons in area 17 of cat visual cortex (König et al., 1995). A systematic variation of the orientation of visual stimuli led to neurons with optimal input, having responses that tended to have a phase lead compared to neurons with suboptimal input. These results are consistent with an interpretation based on our results in which the suboptimally stimulated neurons have fewer active afferents and therefore take longer (relative to optimally stimulated neurons) to reach threshold.

This investigation has highlighted the role of the threshold in relation to the number and amplitude of the synaptic inputs in describing the distribution of output spikes. We have studied an idealized situation in which spontaneous activity is neglected, and investigations are currently underway to analyze the integrate-and-fire model with Poisson distributed inputs using methods similar to those presented above (Burkitt \& Clark, 1998a). This will enable the study of more complex systems of synaptic inputs involving partial synchronization, together with spontaneous activity or systematic phase delays, such as occur in auditory nerve fibers excited by a traveling 


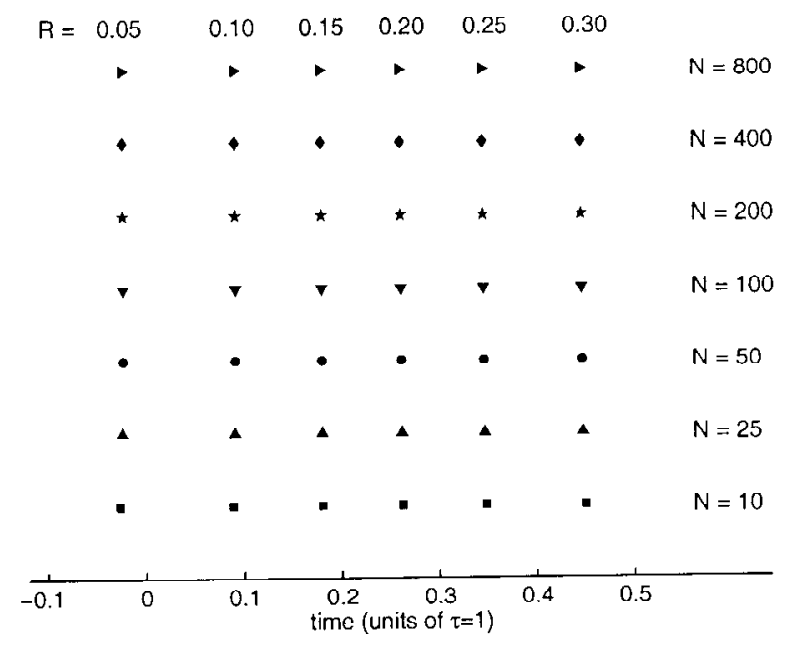

Figure 12: Dependence of the average time of spiking (i.e., center of the spiking distribution) on the number of inputs $N$ and the threshold ratio, for the generalized leaky integrate-and-fire model with $\alpha=5$ and $\sigma_{\text {in }}=0.2$. The different symbols correspond to different numbers of inputs, given on the right of the figure. The results for each value of the threshold ratio $R$ (given at the top of the figure) show only a small variation in time over the entire set of input values $N$. Time is measured in units of $\tau$, with the center of the incoming distribution of EPSPs at $t=0$.

wave along the basilar membrane of the inner ear (Bruce, Irlicht, \& Clark, 1998).

In conclusion, we have presented a new technique for analyzing integrateand-fire neurons with inputs that are synchronized (with some temporal jitter). The results are highly accurate in the physiologically interesting domain in which a threshold unit sums a large number of small-amplitude postsynaptic potentials. The technique allows us to investigate models with arbitrary postsynaptic response functions. The results for the analysis of synchronization in three classes of the integrate-and-fire model agree with both known analytic results and numerical simulations. In a layered network, the dramatic reduction in jitter that is observed in these neural models represents a balance between the various sources of input jitter (the variation in thresholds of the neurons, their number of active inputs, propagation times, and their synaptic jitter) and the large convergence of inputs that tends to reduce the output jitter. 


\section{Appendix A: Evaluating the Probability Distribution}

The probability distribution is evaluated as follows:

$$
\begin{aligned}
& \operatorname{Pr}\left\{V(t) \geq v \mid V(-\infty)=v_{0}\right\} \\
& =\int_{v-v_{0}}^{\infty} \frac{d \lambda}{2 \pi} \int_{-\infty}^{\infty} d x \exp \{i x \lambda+N \ln F(x, t)\} \\
& =\int_{v-v_{0}}^{\infty} \frac{d \lambda}{2 \pi} \int_{-\infty}^{\infty} d x \exp \left\{i x \lambda-i x N a D(t)-\frac{x^{2}}{2} N a^{2}\left[E(t)-D^{2}(t)\right]\right\}
\end{aligned}
$$

where the term $\ln F(x, t)$ has been expanded using the standard Taylor's series expansion. The $x$-integral is a gaussian integral that is evaluated by completing the square, and the $\lambda$-integral likewise becomes a gaussian integral,

$$
\begin{aligned}
\operatorname{Pr}\left\{V(t) \geq v \mid V(-\infty)=v_{0}\right\} & =\int_{v-v_{0}}^{\infty} \frac{d \lambda}{\sqrt{2 \pi \Gamma(t)}} \exp \left\{-\frac{(\lambda-\Lambda(t))^{2}}{2 \Gamma(t)}\right\} \\
& =\frac{1}{2}\left[1-\operatorname{erf}\left(\frac{v-v_{0}-\Lambda(t)}{\sqrt{2 \Gamma(t)}}\right)\right],
\end{aligned}
$$

where $\Lambda(t)$ and $\Gamma(t)$ are given by equation 2.10 .

\section{Appendix B: Evaluating the Joint Probability Density}

The joint probability density is evaluated (for $t_{1}<t_{2}$ ) as

$$
\begin{aligned}
& p\left(v_{2}, t_{2}, v_{1}, t_{1} \mid v_{0}\right) \\
& =\prod_{k}^{N} \int_{-\infty}^{\infty} d t_{k} p\left(t_{k}\right) \delta\left(V\left(t_{1}\right)-v_{1}\right) \delta\left(V\left(t_{2}\right)-v_{2}\right) \\
& =\int_{-\infty}^{\infty} \frac{d x_{2}}{2 \pi} \int_{-\infty}^{\infty} \frac{d x_{1}}{2 \pi} \exp \left\{i x_{2}\left(v_{2}-v_{0}\right)+i x_{1}\left(v_{1}-v_{0}\right)\right. \\
& \left.+N \ln F\left(x_{2}, x_{1}, t_{2}, t_{1}\right)\right\}
\end{aligned}
$$

$F\left(x_{2}, x_{1}, t_{2}, t_{1}\right)$ contains cross-terms in $x_{2} x_{1}$,

$$
\begin{aligned}
F\left(x_{2}, x_{1}, t_{2}, t_{1}\right)= & 1-i x_{2} a D\left(t_{2}\right)-i x_{1} a D\left(t_{1}\right) \\
& -\frac{x_{2}^{2}}{2} a^{2} E\left(t_{2}\right)-\frac{x_{1}^{2}}{2} a^{2} E\left(t_{1}\right)-x_{2} x_{1} a^{2} G\left(t_{2}, t_{1}\right),
\end{aligned}
$$

where $D(t), E(t)$ are given by equation 2.8 and $G\left(t_{2}, t_{1}\right)$ by equation 2.17 . The term $\ln F\left(x_{2}, x_{1}, t_{2}, t_{1}\right)$ is expanded in the amplitude of the synaptic response function as before, and the cross-term $x_{2} x_{1}$ is eliminated by the change of 
variable $x_{1} \rightarrow x_{1}+\kappa\left(t_{2}, t_{1}\right) x_{2}$ where $\kappa\left(t_{2}, t_{1}\right)$ is defined in equation 2.16 . The $x_{1}$ - and $x_{2}$-integrals are now independent and may be evaluated. The $x_{1}$ integral yields exactly $p\left(v_{1}, t_{1} \mid v_{0}\right)$, and the $x_{2}$-integral gives the conditional probability density (see equation 2.15).

\section{Acknowledgments}

This work was funded by the Cooperative Research Centre for Cochlear Implant, Speech, and Hearing Research.

\section{References}

Abbott, L. F. (1994). Decoding neuronal firing and modelling neural networks. Quarterly Review of Biophysics, 27, 291-331.

Abeles, M. (1982). Local cortical circuits: An electrophysiological study. Berlin: Springer-Verlag.

Abeles, M. (1991). Corticonics: Neural circuits of the cerebral cortex. New York: Cambridge University Press.

Adrian, E. (1928). The basis of sensation: The action of sense organs. London: Christophers.

Bernander, O., Koch, C., \& Usher, M. (1994). The effect of synchronized inputs at the single neuron level. Neural Comp., 6, 622-641.

Bialek, W., \& Rieke, F. (1992). Reliability and information transmission in spiking neurons. Trends Neurosci., 15, 428-434.

Bialek, W., Rieke, F., de Ruyter van Steveninck, R. R., \& Warland, D. (1991). Reading a neural code. Science, 252, 1854-1857.

Bruce, I. C., Irlicht, L. S., \& Clark, G. M. (1998). A mathematical analysis of spatiotemporal summation of auditory nerve fibers. Information Sciences, 111, 303-334.

Burkitt, A. N., \& Clark, G. M. (1998a). Calculation of interspike intervals for integrate and fire neurons with Poisson distribution of synaptic inputs. Unpublished manuscript.

Burkitt, A. N., \& Clark, G. M. (1998b). Manuscript in preparation.

Clark, G. M. (1996). Electrical stimulation of the auditory nerve: The coding of frequency, the perception of pitch and the development of cochlear implant speech processing strategies for profoundly deaf people. Clinical and Experimental Pharmacology and Physiology, 23, 766-776.

deCharms, R. C., \& Merzenich, M. M. (1996). Primary cortical representation of sounds by the coordination of action-potential timing. Nature, 381, 610-613.

Diesmann, M., Gewaltig, M. O., \& Aertsen, A. (1996). Characterization of synfire activity by propagating "pulse packets." In J. Bower (Ed.), Computational neuroscience: trends in research (pp. 59-64). San Diego: Academic Press.

Eckhorn, R., Bauer, R., Jordan, W., Brosch, M., Kruse, W., Munk, M., \& Reitboeck, H. J. (1988). Coherent oscillations: A mechanism of feature linking in the visual cortex? Biol. Cybern, 60, 121-130. 
Engel, A. K., König, P., Kreiter, A. K., Schillen, T. B., \& Singer, W. (1992). Temporal coding in the visual cortex: New vistas on integration in the nervous system. Trends Neurosci., 15, 218-226.

Engel, A. K., König, P., \& Singer, W. (1991). Direct physiological evidence for scene segmentation by temporal coding. Proc. Natl. Acad. Sci. USA, 88, 91369140.

Gabbiani, F., and Koch, C. (1996). Coding of time-varying signals in spike trains of integrate-and-fire neurons with random threshold. Neural Comp., 8, 44-66.

Gerstein, G. L., \& Mandelbrot, B. (1964). Random walk models for the spike activity of a single neuron. Biophys. J., 4, 41-68.

Gerstner, W. (1995). Time structure of the activity in neural network models. Phys. Rev. E, 51, 738-758.

Gerstner, W., \& van Hemmen, J. L. (1996). What matters in neuronal locking? Neural Comp., 8, 1653-1676.

Gluss, B. (1967). A model for neuron firing with exponential decay of potential resulting in diffusion equations for probability density. Bull. Math. Biophysics, 29, 233-243.

Hodgkin, A. L. and Huxley, A. F. (1952). A quantitative description of membrane current and its application to conduction and excitation in nerve. J. Physiol. (London), 117, 500-544.

Hopfield, J. J. (1995). Pattern recognition computation using action potential timing for stimulus representation. Nature, 376, 33-36.

Jack, J. J. B., Noble, D., \& Tsien, R. W. (1985). Electric current flow in excitable cells. Oxford: Clarendon.

Johnson, D. H. (1980). The relationship between spike rate and synchrony in responses to auditory-nerve fibers to single tones. J. Acoust. Soc. Am., 68, $1115-1122$.

Joris, P. X., Carney, L. H., Smith, P. H., \& Yin, T. C. T. (1994). Enhancement of neural synchronization in the anteroventral cochlear nucleus. I. Responses to tones at the characteristic frequency. J. Neurophysiol., 71, 1022-1036.

Judd, K. T., and Aihara, K. (1993). Pulse propagation networks: A neural network model that uses temporal coding by action potentials. Neural Networks, 6, 203-215.

Kistler, W. M., Gerstner, W., \& van Hemmen, J. L. (1997). Reduction of the Hodgkin-Huxley equations to a single-variable threshold model. Neural Comp., 9, 1015-1045.

König, P., Engel, A. E., Roelfsema, P. R., \& Singer, W. (1995). How precise is neuronal synchronization? Neural Comp., 7, 469-485.

Lapicque, L. (1907). Recherches quantitatives sur l'excitation électrique des nerfs traitée comme une polarization. J. Physiol. (Paris) 9, 620-635.

Maass, W. (1996a). Lower bounds for the computational power of networks of spiking neurons. Neural Comp., 8, 1-40.

Maass, W. (1996b). Networks of spiking neurons. In P. Bartlett, A. Burkitt, \& R. C. Williamson (Eds.), Proceedings of the Seventh Australian Conference on Neural Networks, Canberra: Australian National University.

Maršálek, P., Koch, C., \& Maunsell, J. (1997). On the relationship between synaptic input and spike output jitter in individual neurons. Proc. Natl. Acad. Sci. USA, 94, 735-740. 
Milner, P. M. (1974). A model for visual shape recognition. Psychol. Rev., 81, 521-535.

Paolini, A. G., Clark, G. M., \& Burkitt, A. N. (1997). Intracellular responses of rat cochlear nucleus to sound and its role in temporal coding. NeuroReport, 8(15), 3415-3422.

Plesser, H. E., \& Tanaka, S. (1997). Stochastic resonance in a model neuron with reset. Phys. Lett. A, 225, 228-234.

Press, W. H., Flannery, B. P., Teukolsky, S. A., \& Vetterling, W. T. (1992). Numerical recipes in Fortran: The art of scientific computing. Cambridge: Cambridge University Press.

Rhode, W. S., \& Smith, P. H. (1986). Encoding timing and intensity in the ventral cochlear nucleus of the cat. J. Neurophysiol., 56, 261-286.

Singer, W. (1993). Synchronization of cortical activity and its putative role in information processing and learning. Annu. Rev. Physiol., 55, 349-374.

Stein, R. B. (1965). A theoretical analysis of neuronal variability. Biophys. J., 5, 173-194.

Tuckwell, H. C. (1988a). Introduction to theoretical neurobiology: Vol. 1, Linear cable theory and dendritic structure. Cambridge: Cambridge University Press.

Tuckwell, H. C. (1988b). Introduction to theoretical neurobiology: Vol. 2, Nonlinear and stochastic theories. Cambridge: Cambridge University Press.

Tuckwell, H. C. (1989). Stochastic processes in the neurosciences. Philadelphia: Society for Industrial and Applied Mathematics.

Usher, M., Schuster, H. G., \& Niebur, E. (1993). Dynamics of populations of integrate-and-fire neurons, partial synchronizaton and memory. Neural Comp., 5, 570-586.

Received November 12, 1997; accepted August 10, 1998. 


\section{University Library}

\section{- M M I N E R VA A gateway to Melbourne's research publications}

Minerva Access is the Institutional Repository of The University of Melbourne

Author/s:

Burkitt, A. N.;Clark, Graeme M.

Title:

Analysis of integrate and fire neurons: synchronization of synaptic input and spike output

Date:

1999

Citation:

Burkitt, A. N., \& Clark, G. M. (1999). Analysis of integrate and fire neurons: synchronization of synaptic input and spike output. Neural Computation, 11(4), 871-901.

Persistent Link:

http://hdl.handle.net/11343/27524 